\title{
Nodal activates smad and extracellular signal-regulated kinases 1/2 pathways promoting renal cell carcinoma proliferation
}

\author{
ZHIWEI ZHANG*, TAO JIANG*, QUANLIN LI, JIANBO WANG, DEYONG YANG, \\ XIANCHENG LI, QIFEI WANG and XISHUANG SONG
}

\begin{abstract}
Department of Urinary Surgery, First Affiliated Hospital of Dalian Medical University, Dalian, Liaoning 116000, P.R. China
\end{abstract}
Received April 18,2014; Accepted January 7, 2015

DOI: $10.3892 / \mathrm{mmr} .2015 .3343$

\begin{abstract}
Expression of the nodal gene is high in a number of tumor cell types and may promote tumor growth. The expression of lefty, an inhibitor of nodal is often reduced in tumor cells. To the best of our knowledge, few studies have investigated the expression of nodal and lefty in renal cell carcinoma (RCC) cells. In the present study, quantitative polymerase chain reaction assays demonstrated that the level of nodal expression in RCC cells was high compared with that of adjacent non-tumor tissue cells, while the opposite pattern was observed for the level of lefty expression. Furthermore, lefty overexpression in RCC cells inhibited the expression of nodal. Nodal overexpression promoted RCC cell proliferation and invasion, and inhibited RCC cell apoptosis. Nodal downregulation and lefty overexpression led to similar observations: The inhibition of RCC cell proliferation and invasion, and the promotion of RCC cell apoptosis. The results of the present study suggested that the expression of nodal promoted RCC growth by activating the smad and extracellular signal-regulated kinases $1 / 2$ pathways. The expression of lefty in RCC cells was lower than that in adjacent non-tumor cells, which may result in the overexpression of nodal, thereby promoting the growth of RCC. The results of the present study may therefore be useful for the development of novel biomarkers for RCC tumor diagnosis, and suggest a potential target gene for the treatment of RCC.
\end{abstract}

\section{Introduction}

Renal cell carcinoma (RCC) is a malignant tumor, which originates in the renal tubular epithelial system. It is one of

Correspondence to: Dr Xishuang Song, Department of Urinary Surgery, First Affiliated Hospital of Dalian Medical University, 222 Zhongshan Road, Xigang, Dalian, Liaoning 116000, P.R. China E-mail: song-xishuang@163.com; ysgene@126.com

*Contributed equally

Key words: extracellular signal-regulated kinases 1/2, nodal, lefty, smad, renal cell carcinoma the most common tumors in the urinary system, and clear cell RCC (CCRCC) is the commonest pathological type of RCC (85-90\%) (1). The incidence of RCC is comparable with that of bladder cancer, and ranks second among urinary tumors, accounting for $\sim 2-3 \%$ of all malignant human tumors (1). The incidence of RCC and the mortality due to RCC is growing, with a yearly increase of the incidence of RCC of $2 \%$ worldwide (2-3). However, the pathogenesis of RCC remains poorly understood. Oncogene activation, and anti-oncogene mutation and inactivation may lead to RCC tumor development. Therefore, further research may help to provide novel methods for tumor prevention and treatment.

Nodal is a member of the transforming growth factor $\beta$ (TGF- $\beta$ ) superfamily. It is involved in the development of embryonic stem cells, via regulation of the induction of embryonic tissues to form a complete axis (4,5). Nodal is a primary element that regulates early embryo-inducing signals, which involves a series of events, such as the formation of the mesoderm and entoderm, the determination of the location of the front-rear axis, and the specialization of the left-right axis. Therefore, nodal exhibits important functions in the early developmental stages of vertebrates (4-5). The nodal signaling pathway involves the secretion of nodal into the cytoplasm, where it binds with the co-receptor cripto-1, which forms a bond with II-type receptor activin receptor type-2B and I-type receptor ALK4/7 (5). This leads to the formation and phosphorylation of the ALK receptor complex, which activate $\operatorname{smad} 2$ and $\operatorname{smad} 3$ expression in the cytoplasm. Subsequently, the smad $2 / 3$ compound and smad4 develop into active trimers, which pass into the nucleus. In the nucleus the smad2/3 compound and smad4 bind with forkhead box protein H1, mix paired-like homeobox, and other transcription factors and co-activators or co-repressors, which regulate the transcription of Nodal responsive genes, such as Lefty (5). Lefty is a member of the TGF $\beta$ superfamily and is an important cytokine. It is involved in the regulation of embryonic development and stem cell differentiation, and its expression inhibits the nodal signaling pathway (6). Lefty inhibits nodal signaling by binding directly with nodal or with cripto, thereby preventing the formation of an active nodal/activin receptor complex (7). A balance between lefty and nodal activity is important for a number of developmental processes, as demonstrated by the severe and often fatal phenotypes observed in lefty- or nodal-deficient embryos (4). 
Nodal protein expression has been reported in malignant melanoma, testicular cancer, breast cancer, and brain glioma. Its expression is closely associated with tumor invasion, metastasis and poor prognosis (8-10). A number of studies have shown that nodal may be developed as a biomarker for monitoring the malignant progression of tumors and as a target molecule for clinical intervention (9). Furthermore, nodal is capable of regulating tumor cell plasticity; the downregulation of nodal expression abolishes tumor cell plasticity, which prevents tumor cells from undergoing vasculogenic mimicry (VM) (9). McAllister et al (11) demonstrated that nodal gene expression is associated with the formation of VM-like structures in a physiological model of human melanoma tumorigenesis, providing further support for an association between nodal expression and the formation of channel-like structures. Studies have demonstrated an association between nodal expression and human malignant melanoma (12). A previous study demonstrated that nodal downregulation may reduce the occurrence of VM in human malignant melanoma cells, induce cell apoptosis, and inhibit the development of tumors (9).

Previous studies have demonstrated that tumor metastasis and embryonic development exhibit a similar activation of nodal signaling pathways (5). However, regulation of the nodal signaling pathways in tumor metastasis and embryonic development is different. Embryonic tissue is capable of secreting the following endogenous nodal signaling pathway inhibitors: I-smads (smad6, smad7), lefty A/B and other proteins that regulate developmental processes. These endogenous nodal signaling pathway inhibitors, however, were not detected in certain invasive tumor cells, such as melanoma cells (13-14). The lack of these endogenous nodal signaling pathway inhibitors in tumor cells may therefore be associated with tumor invasion and metastasis. Studies have shown that the embryonic microenvironment, in particular the microenvironment of embryonic stem cells, may enable tumor cells to obtain more differentiated phenotypes and to markedly reduce the malignancy grade $(12,13)$. Cucina et al $(15)$ exposed metastatic melanoma cells to an embryonic microenvironment prior to zebrafish gastrulation, which led to gene rearrangement and the formation of non-oncogenic phenotypes. Metastatic melanoma cells transplanted into developing chick embryos are capable of following the neural crest migration pathway. The cells therefore lose tumorigenicity and exhibit a phenotype similar to that of healthy neural crest cells (16). A high expression of nodal in migrating melanoma cells and breast cancer cells may inhibit cell differentiation, whereas the presence of glycosylated lefty in the embryonic stem cell microenvironment may inhibit nodal expression, thereby reducing the degree of malignancy of tumor cells $(15,16)$. Previous research has shown that the extraction of a small concentration of lefty $(20-50 \mathrm{ng} / \mathrm{ml})$ from human embryonic stem cells (hESCs) is capable of reducing melanoma C8161 cell proliferation and increasing apoptosis, leading to reduced tumor invasiveness (14). Therefore, restoring the balance of the nodal signaling pathway may aid in the control of tumor cell proliferation.

To the best of our knowledge, few studies have investigated the role of lefty and nodal in RCC. The present study examined whether the expression of lefty and nodal in RCC cells is similar to that of other types tumor cells. The regulatory mechanisms underlying lefty and nodal expression in RCC cells were also investigated.

\section{Materials and methods}

Tissue samples and cell culture. Tumor and adjacent non-tumor tissues (45 pairs) were obtained from patients with CCRCC. The tissue samples had been resected at the First Affiliated Hospital of Dalian Medical University (Dalian, China) between February 2012 and April 2013. The patients were of Chinese origin and they fulfilled the RCC criteria of the World Health Organization (WHO; 17). Tissue samples were subjected to pathological examinations in order to confirm the diagnosis of RCC. RCC staging was assessed using the TNM staging system for kidney cancer revised by the American Joint Committee On Cancer (AJCC; 18). The present study was approved by the ethics committee of First Affiliated Hospital of Dalian Medical University. All patients gave informed written consent prior to the initiation of the study. The demographic data and clinicopathological features of the patients are summarized in Table I. Tissue samples were snap-frozen in liquid nitrogen immediately following resection, and stored at $-80^{\circ} \mathrm{C}$. Human A498 and 786-O cell lines were obtained from the American Type Culture Collection (Rockville, MD, USA) and cultured in high glucose Dulbecco's modified Eagle's medium (GE Healthcare Life Sciences, Beijing, China) with $10 \%$ fetal bovine serum (Gibco, China) in $<5 \% \mathrm{CO}_{2}$ at $37^{\circ} \mathrm{C}$.

Lefty and nodal overexpression vector construction and small interfering RNA (siRNA) design. RCC cells and hESCs were collected in order to extract RNA, using TRIzol ${ }^{\circledR}$ (Invitrogen Life Technologies, Carlsbad, CA, USA) according to the manufacturer's instructions. Subsequently, reverse transcription polymerase chain reaction (RT-PCR) was conducted in order to amplify the coding regions of lefty and nodal. The products were digested with Kpn I and EcoR I (Takara Bio, Inc., Shiga, Japan), cloned into pcDNA3.1 vectors (Promega, Beijing, China) sequenced and verified using ABI3730xl DNA Analyzer (Applied Biosystems, Forster City, CA, USA). The following primers were used for PCR: Forward: 5'-GGG GTACCGCCACCATGCAGCCCCTGTGGCTC-3' and reverse: 5'-CGGAATTCCTATGGCTGGAGCCTCCTTG-3' for lefty, forward: 5'-GGGGTACCGCCACCATGCAC GCCCACTGCCTG-3' and reverse: 5'-CGGAATTCTCAG AGGCACCCACATTCTTC-3' for nodal, forward: 5'-AGA CAUGAUCGUGGAAGAATT-3' and reverse: 5'-UUC UUCCACGAUCAUGUCUTT-3' for nodal siRNA (19), forward: 5'-CUGUGUGAGUUCGCCUUCAUUTT-3', and reverse: 5'-UGAAGGCGAACUCACACAGUUTT-3' for smad3 siRNA (20) and forward: 5'-UUCUCCGAAC GUGUCACGUTT-3' and reverse: 5'-ACGUGACACGU UCGGAGAATT-3' for control siRNA, which were taken from a previous publication (19).

Transfection and signaling pathway inhibition. Cells $\left(1 \times 10^{5}\right.$ cells $\left./ \mathrm{ml}\right)$ were seed into 6 -well plates and incubated for $24 \mathrm{~h}$ at $37^{\circ} \mathrm{C}$. Once cells had reached $\sim 70 \%$ confluence, plasmid and siRNA transfection were conducted using Lipofectamine $2000^{\circledR}$ according to the manufacturer's instructions (Invitrogen Life Technologies). Following 4-6 h of transfection the medium was changed. Extracellular signal-related kinase (Erk) inhibitor II was added to 786-O cells post transfection (FR180204; $10 \mu \mathrm{M}$; Santa Cruz Biotechnology, Inc., Dallas, 
Table I. Clinicopathological parameters of patients with renal cell carcinoma $(n=45)$.

\begin{tabular}{lr}
\hline Characteristic & Frequency $(\%)$ \\
\hline Gender & $23(51.1)$ \\
Male & $22(48.9)$ \\
Female & \\
Age (years) & $7(15.6)$ \\
$\leq 50$ & $38(84.4)$ \\
$>50$ & \\
Size of tumor (length, cm) & $27(60.0)$ \\
$\leq 7$ & $18(40.0)$ \\
$>7$ & \\
TNM staging & $15(33.3)$ \\
I & $12(26.7)$ \\
II & $14(31.1)$ \\
III & $4(8.9)$ \\
IV & \\
Fuhrman grade & $27(60.0)$ \\
High differentiation & $14(31.1)$ \\
Moderate differentiation & $4(8.9)$ \\
Poor differentiation & \\
\hline
\end{tabular}

TX, USA). Following $24 \mathrm{~h}$ of transfection, all cells were cultured for a further $24-48 \mathrm{~h}$ at $37^{\circ} \mathrm{C}$.

$R T-q P C R$. Total RNA was extracted from the RCC cells and hESCs using TRIzol (Invitrogen Life Technologies). The concentration of extracted total RNA was determined by measuring the absorbance at $260 \mathrm{~nm}$ using a Cary 8454 UV-Visible Spectrophotometer . Total RNA (1 $\mu \mathrm{g})$ was used for first-strand cDNA synthesis using RevertAid ${ }^{\mathrm{TM}}$, a first strand cDNA Synthesis kit (Fermentas ${ }^{\mathrm{TM}}$, Logan, UT, USA). quantitative PCR (qPCR) was performed on $100 \mathrm{ng}$ of cDNA in $20 \mu \mathrm{l}$ of reaction mixture using SYBR Premix Ex Taq (Takara). The following primers were used for PCR: Forward: 5'-GCGAGTGTCCTAATCCTGTTG-3' and reverse: 5'-CAGCGGCTTGGTCTTCAC-3' for nodal-QT, forward: 5'-AACCGCACCTCCCTCATC-3' and reverse: 5'-GCTGCTGCCAGAAGTTCAC-3' for lefty-QT and forward: 5'-GGTATCGTGGAAGGACTC-3' and reverse: 5'-GTAGAGGCAGGGATGATG-3' for glyceraldehyde 3-phosphate dehydrogenase (GAPDH). The following PCR protocol was performed: One cycle of $95^{\circ} \mathrm{C}$ for $5 \mathrm{~min}$, and 40 cycles of $95^{\circ} \mathrm{C}$ for $30 \mathrm{sec}, 55^{\circ} \mathrm{C}$ for $30 \mathrm{sec}$ and $72^{\circ} \mathrm{C}$ for $30 \mathrm{sec}$. Three independent experiments were conducted for each sample. Data were analyzed by comparing the $2^{-\Delta \Delta \mathrm{Ct}}$ values.

Western blotting. Total cellular proteins were extracted by incubating cells in lysis buffer (Pierce Biotechnology, Inc., Rockford, IL,USA) The protein concentrations in the cell lysates were determined using a bicinchoninic acid assay (Pierce Biotechnology, Inc.). SDS-PAGE was conducted using $8 \%$ glycine gels (Bio-Rad, Hercules, CA, USA) loading equal quantities of proteins $(20 \mu \mathrm{g})$ per lane. Following electrophoresis, separated protein bands were transferred to a nitrocellulose membrane (Pierce Biotechnology, Inc.) and blocked using 5\% non-fat milk in tris-buffered saline with Tween-20 buffer for $1 \mathrm{~h}$. Subsequently, the membranes were incubated with rabbit polyclonal immunoglobulin (Ig)G anti-nodal (sc-28913; Santa Cruz Biotechnology, Inc.; 1:300), rabbit polyclonal anti-lefty (ab30955; Abcam, Cambridge, UK; 1:500), rabbit polyclonal anti-smad2/smad3 (cat. no. 3102; Cell Signaling Technology, Inc., Danvers, MA, USA; 1:600), rabbit monoclonal anti-phospho-Smad2/Smad3 (cat. no. 8828; Cell Signaling Technology, Inc.; 1:500), rabbit monoclonal anti-Erk1/2 (cat. no. 4695; Cell Signaling Technology, Inc.; 1:800), rabbit monoclonal anti-phospho-Erk1/2 (cat. no. 4370; Cell Signaling Technology, Inc.; 1:600), and rabbit polyclonal anti-GAPDH (NBP1-47339; Novus Biologicals, Littleton, CO, USA; 1:1,000) antibodies overnight at $4^{\circ} \mathrm{C}$. Subsequently, goat anti-rabbit IgG secondary antibodies conjugated with horseradish peroxidase (cat. no. 7074; Cell Signaling Technology, Inc.; 1:7,000-8,000) were incubated with the membranes for $1 \mathrm{~h}$ at room temperature. Protein bands were detected using ECL color (Pierce Biotechnology, Inc.).

Cell proliferation. Cell proliferation was quantified using a Bromodeoxyuridine colorimetric immunoassay kit (Cell Proliferation ELISA, Roche Diagnostics, Basal, Switzerland), according to the manufacturer's instructions. Cell proliferation was expressed as the mean percentage of cell proliferation of control cells (set at $100 \%$ ).

Annexin-V-FLUOS apoptosis analysis. Following transfection for $72 \mathrm{~h}$, cells were collected. The translocation of phosphatidylserine, a positive cell surface marker for apoptosis, was detected in treated cells using the Annexin-V-FLUOS staining kit (Roche Applied Science, Penzberg, Germany). Cells were suspended in $500 \mu \mathrm{l}$ of binding buffer (Roche Applied Science) and incubated at room temperature in darkness for $15 \mathrm{~min}$. Cells were then labeled with Annexin V-fluorescein isothiocyanate (5 $\mu \mathrm{l}$; Roche Applied Science) and propidium iodide $(5 \mu \mathrm{l})$. The stained cells were then analyzed using flow cytometry (Beckman Coulter, Inc., Brea, CA, USA).

Transwell Matrigel ${ }^{T M}$ invasion assay. Cell invasion was measured using a Transwell Matrigel invasion assay (BD Biosciences, Shanghai, China). Following transfection, cells $\left(200 \mu \mathrm{l} ; 1 \times 10^{6} / \mathrm{ml}\right)$ and complete medium $(600 \mu \mathrm{l})$ were added to the upper and lower compartments of the chamber respectively. Following $48 \mathrm{~h}$ of incubation, cells that had migrated to the lower side of the filter were fixed with $4 \%$ paraformaldehyde (BD Biosciences) for $15 \mathrm{~min}$ at room temperature, washed with phosphate-buffered saline (BD Biosciences), stained with crystal violet (BD Biosciences) and observed under a confocal microscope (Olympus Corp., Beijing, China).

Statistical analysis. Experiments were repeated at least three times and results are expressed as the mean \pm standard deviation. SPSS Inc. (13.0; Chicago, IL, USA) was used for statistical analysis. The differences between two groups were analyzed using two-tailed Student's t-test and the differences between three or more groups were analyzed using one-way analysis of variance. In all cases $\mathrm{P}<0.05$ was considered to indicate a statistically significant difference. 

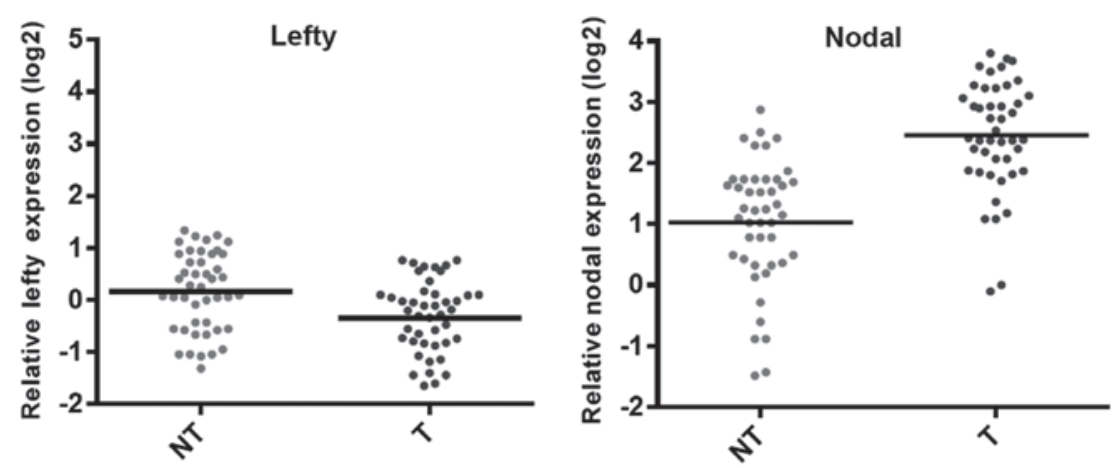

Figure 1. Reverse transcription-quantitative polymerase chain reaction analysis showed downregulation of lefty and upregulation of nodal mRNA expression in patients with renal cell carcinoma. NT, non-tumorous cells; T, tumorous cells.
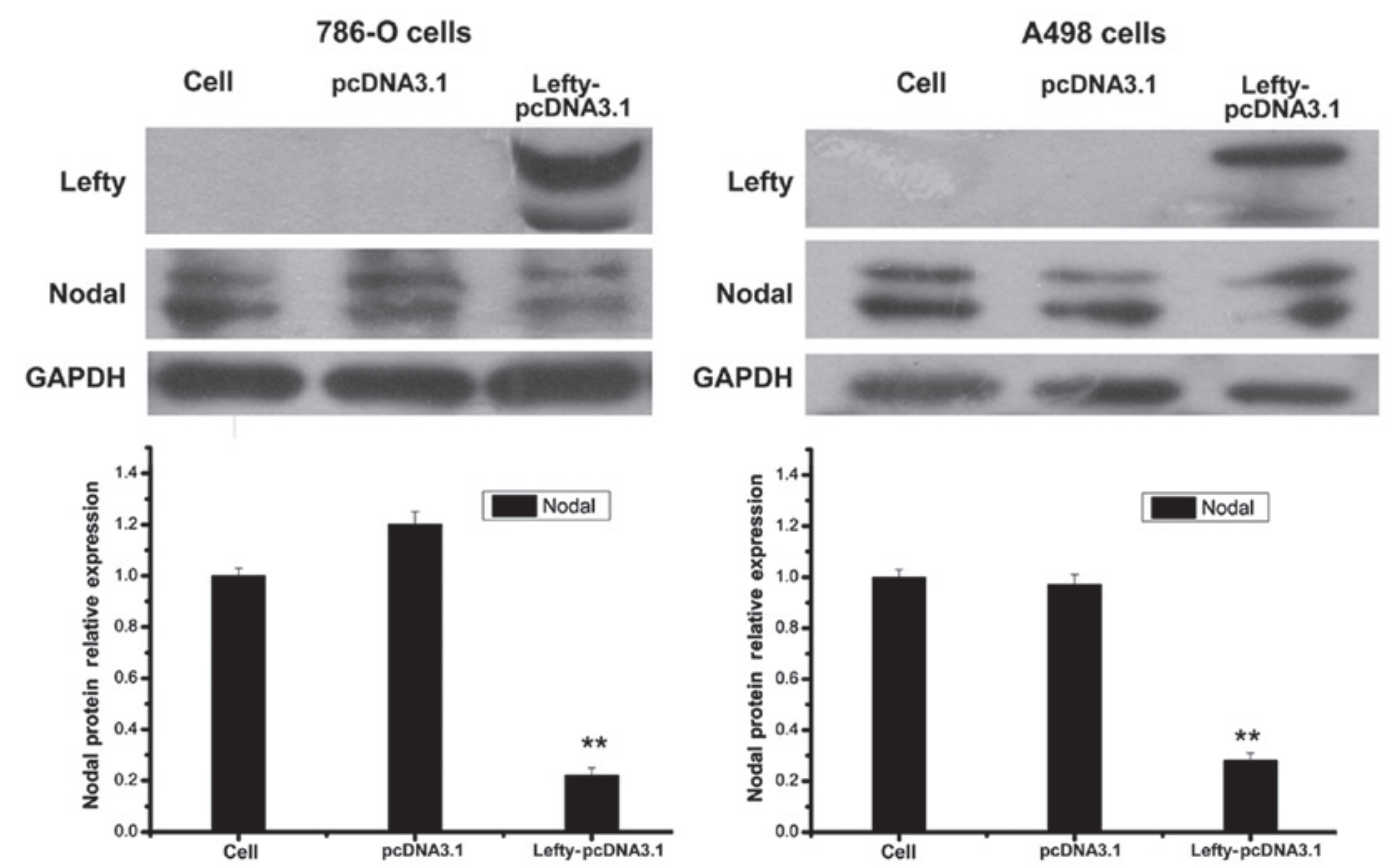

Figure 2. Expression of lefty and nodal in renal cell carcinoma cells. Human 786-O and A498 RCC cells were transfected either with pcDNA3.1 or lefty-pcDNA3.1 (overexpressing lefty). Lefty and nodal mRNA expression levels were measured using western blot analysis. Bars represent the mean \pm standard deviation from three samples. " $\mathrm{P}<0.05$ vs. the control and ${ }^{* *} \mathrm{P}<0.01$ vs. the control. GAPDH, glyceraldehyde 3-phosphate dehydrogenase; Cell, control group.
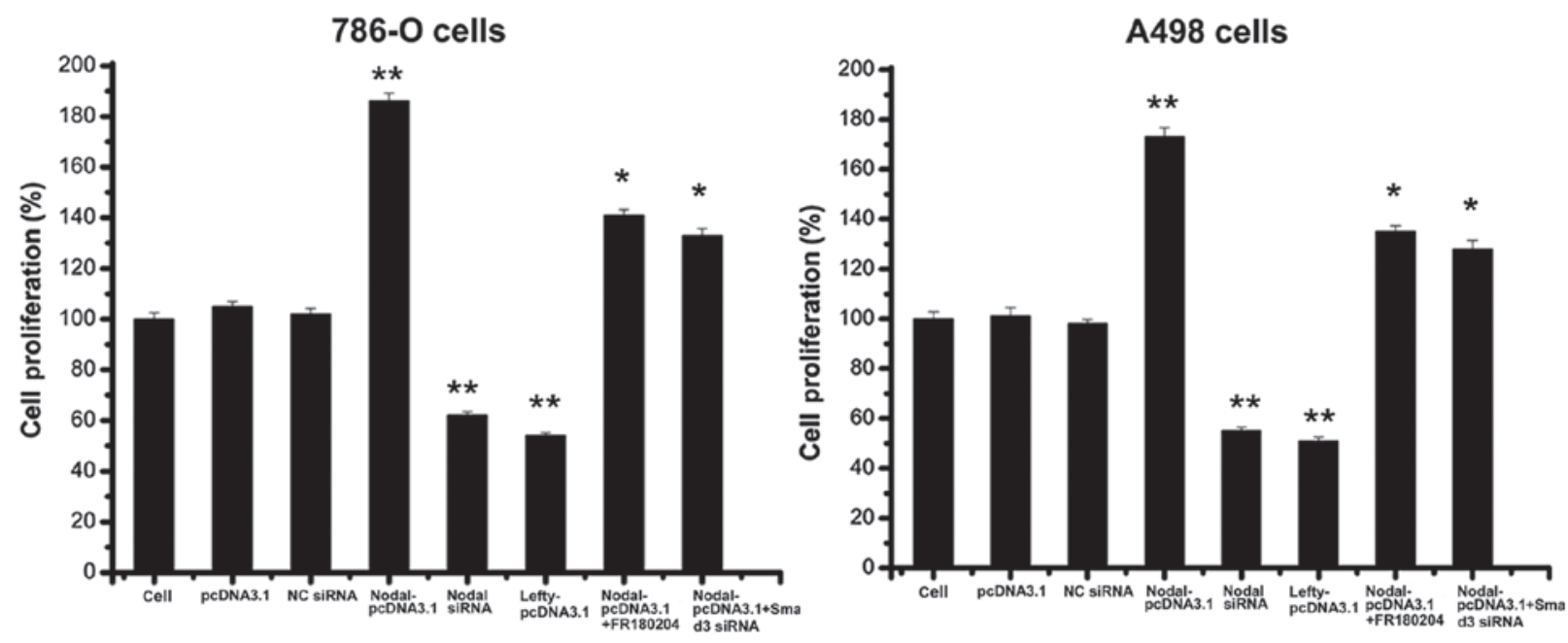

Figure 3. Effect of lefty and nodal expression on renal cell carcinoma cell proliferation. Following treatment with small interfering RNA and/or vector transfection for $24 \mathrm{~h}$, FR 180204 (ERK Inhibitor II) was added to one group of cells. All cells were cultured for a further $48 \mathrm{~h}$. Cell proliferation was measured using a bromodeoxyuridine assay. The results are presented as the mean \pm standard deviation. ${ }^{*} \mathrm{P}<0.05$ vs. control and ${ }^{* *} \mathrm{P}<0.01$ vs. control. NC, non cancerous. Cell, control group. 

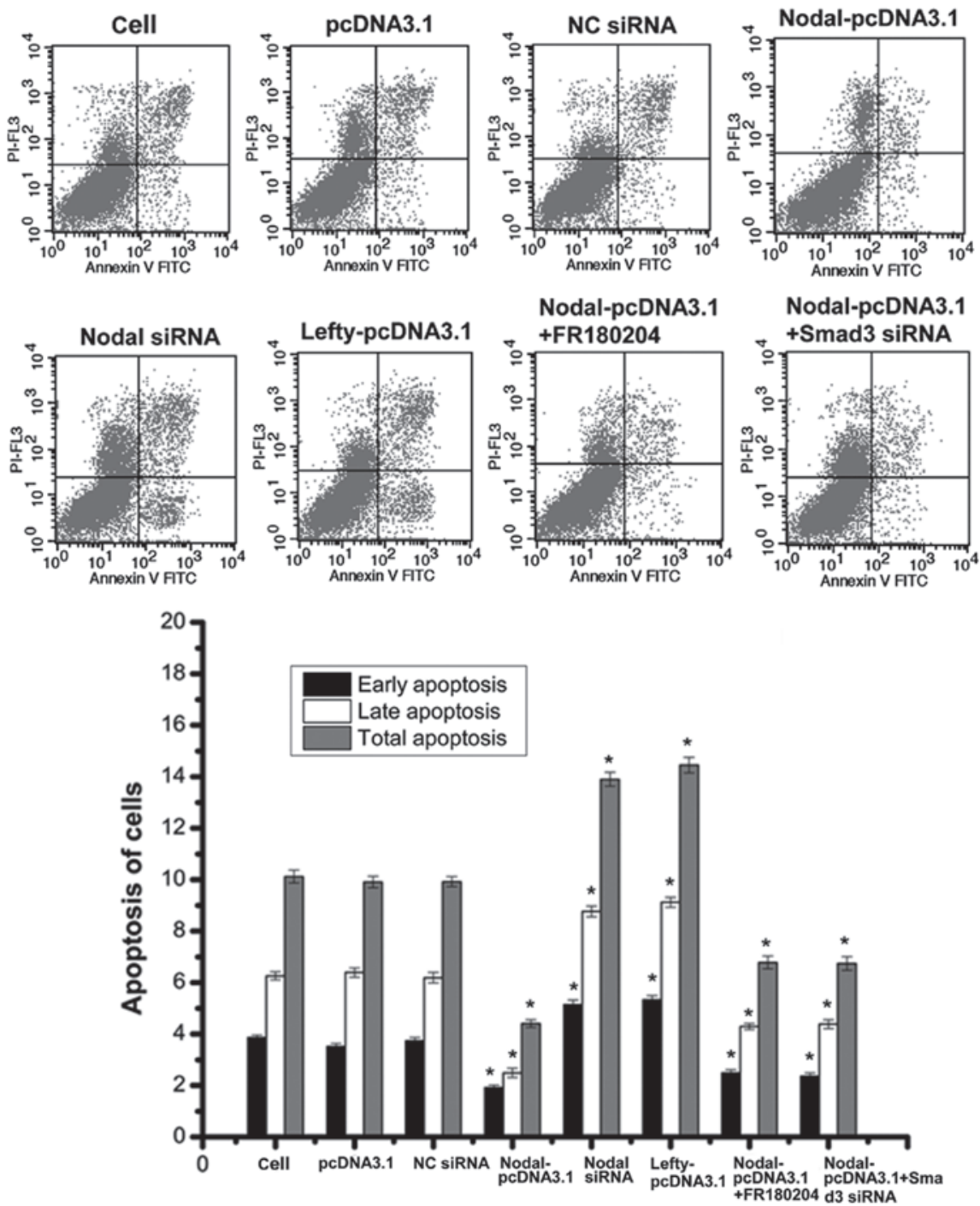

Figure 4. Effect of lefty and nodal expression on 786-O renal cell carcinoma cell apoptosis. 786-O cell apoptosis was measured using Annexin V-fluorescein isothiocyanate propidium iodide flow cytometry analysis. The results are presented as mean \pm standard deviation $(\mathrm{n}=3),{ }^{*} \mathrm{P}<0.05$ vs. the control and ${ }^{* * *} \mathrm{P}<0.01 \mathrm{vs}$. the control. siRNA, small interfering RNA; Cell, control group.

\section{Results}

Expression of lefty and nodal in RCC cells. An RT-PCR analysis of lefty and nodal expression in RCC tumor and adjacent non-tumor cells (45 pairs) was conducted. The results indicated that the expression of nodal in RCC cells was high compared with that in adjacent non-tumor cells (Fig. 1). However, the expression of lefty in RCC was significantly decreased compared with that of the adjacent non-tumor cells $(\mathrm{P}<0.01)$ (Fig. 1). Expression of nodal was significantly lower in RCC cells overexpressing lefty compared with that in the control cells (Fig. 2). These results suggested that lefty expression was lower in RCC cells compared with that in control cells, which may result in a loss of nodal regulation.

Effect of lefty and nodal expression on cell proliferation and apoptosis. Nodal expression promotes cell proliferation and inhibits apoptosis in different types of tumor cells (21-24), and lefty expression results in the opposite effects (25-26). The findings of the present study suggested that nodal overexpression may promote RCC cell proliferation and inhibit apoptosis (Figs. 3 and 4). The downregulation of nodal and the overexpression of lefty led to similar observations: RCC cell proliferation was inhibited and apoptosis was promoted (Figs. 3 and 4). Therefore, the growth of RCC cells may be promoted by nodal expression and inhibited by lefty expression.

Effect of lefty and nodal on cell invasion. Studies have shown that nodal expression is high in metastatic melanoma cell lines (C8131, WM278, and 1205Lu), whereas that of a non-invasive melanoma cell line (C81-61) was shown to be low or defective (27-28). Therefore, nodal expression may promote tumor cell invasion and metastasis. A transwell assay was used to determine the effect of lefty and nodal on RCC cell invasion. The results of the present study demonstrated that the overexpression of nodal promoted RCC cell invasion, whereas RCC cell invasion was inhibited through nodal gene knockdown or lefty overexpression (Fig. 5).

Nodal expression activates smad and ERK1/2 pathways, promoting RCC growth. Nodal is a member of the superfamily TGF- $\beta$, which may activate the smad pathway (5). Therefore, 
A
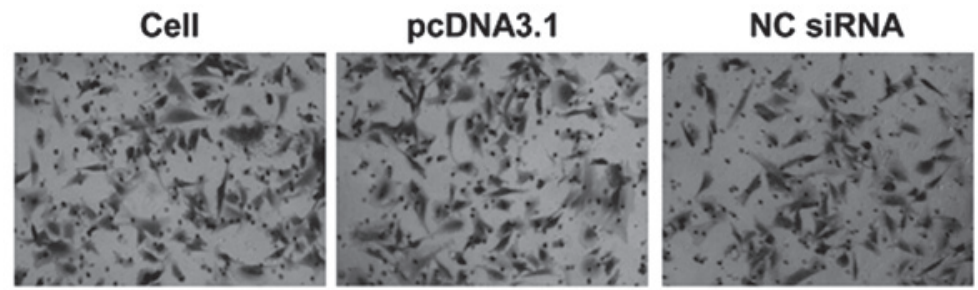

Nodal-pcDNA3.1
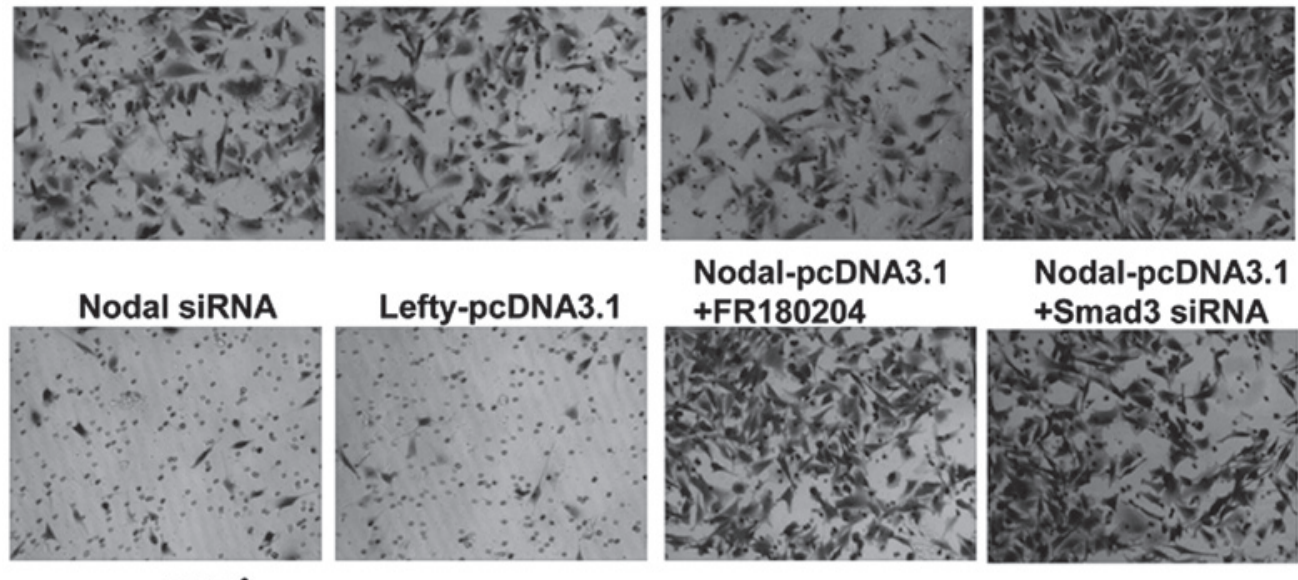

+FR180204

Nodal-pcDNA3.1

+Smad3 SiRNA

B

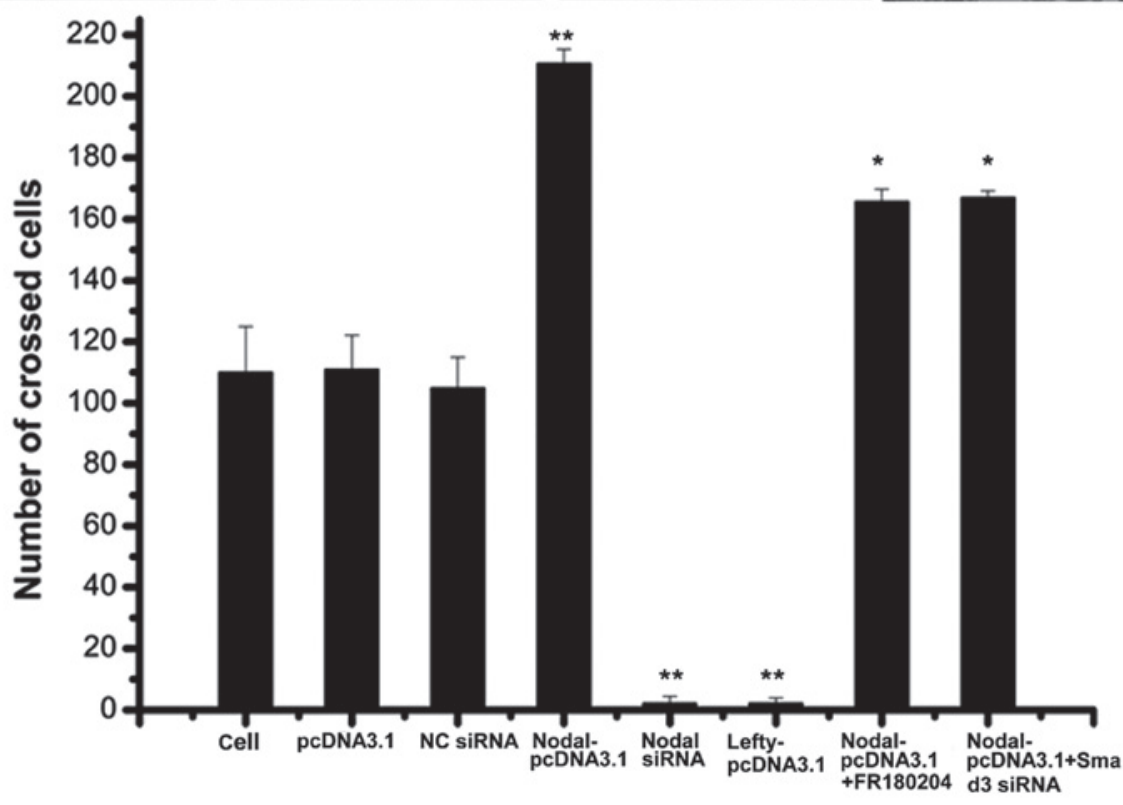

Figure 5. Effect of lefty and nodal expression on 786-O renal cell carcinoma cell invasion. (A) 786-O cell invasion as measured using a Transwell assay. (B) Quantitative analysis of the invasion effects of Lefty and Nodal. Each bar represents the mean \pm standard deviation from three samples. "P $<0.05$ vs. control and ${ }^{* *} \mathrm{P}<0.01$ vs control. NC, non cancerous; siRNA, small interfering RNA; Cell, control group.
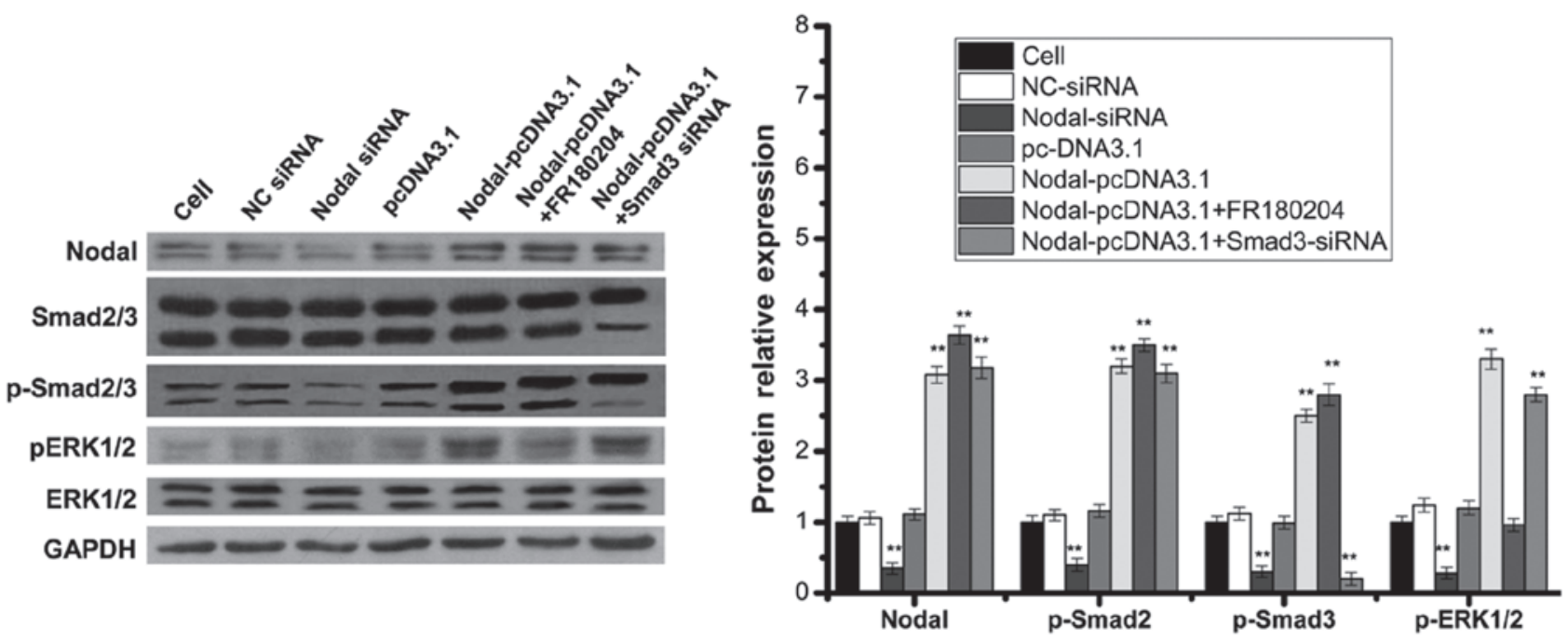

Figure 6. Nodal expression activated the smad and ERK1/2 pathways. 786-O cells were transfected either with NC siRNA, nodal siRNA, pcDNA3.1, nodal overexpression vector without FR180204 (ERK Inhibitor II), nodal overexpression vector with FR180204 or nodal overexpression vector with smad3 siRNA. The expression of nodal, smad2/3, p-smad2/3, ERK1/2 and p-ERK1/2 was measured using western blot analysis. Each bar represents the mean \pm standard deviation from three samples. ${ }^{*} \mathrm{P}<0.05$ vs. control and ${ }^{* *} \mathrm{P}<0.01$ vs control. NC, non-cancerous; siRNA, small interfering RNA; ERK; extracellular signal-regulated kinase; GAPDH, glyceraldehyde 3-phosphate dehydrogenase. 
following the overexpression or downregulation of nodal, the expression of a principle signal transduction molecule involved in the smad pathway, smad2/3, was measured using western blotting. The results demonstrated that nodal overexpression in 786-O RCC cells induced smad2/3 phosphorylation (Fig. 6). By contrast, nodal expression knockdown reduced smad2/3 phosphorylation in 786-O RCC cells (Fig. 6). Therefore, nodal expression may activate the smad pathway. However, following the downregulation of smad3 in RCC cells overexpressing nodal, cell proliferation and invasion was only partly reduced compared with that of cells without smad downregulation. Furthermore, RCC cell apoptosis was not significantly higher in RCC cells overexpressing nodal with smad3 downregulation, compared with cells without smad downregulation (Figs. 3-5). These results suggested that nodal may be involved in other pathways that promote the growth of RCC. In pancreatic cancer cells, the ERK1/2 pathway may inhibit lefty expression induced by TGF- $\beta$ (29). Therefore, nodal may be involved in the ERK1/2 pathway. In the present study, overexpression of nodal promoted ERK1/2 phosphorylation, whereas the downregulation of nodal reduced ERK1/2 phosphorylation (Fig. 6), indicating that nodal may activate the ERK1/2 pathway. Following the addition of FR180204, an ERK inhibitor, RCC cells overexpressing nodal did not exhibit significantly lower levels of RCC cell proliferation and invasion compared with cells overexpressing nodal that did not receive treatment with FR180204. Furthermore, RCC cell apoptosis were not significantly higher in cells overexpressing nodal that were treated with FR180204, compared with those that were not treated with FR180204 (Figs. 3-5). Therefore nodal expression may activate the smad and ERK1/2 pathways, and promote RCC cell proliferation and invasion, in addition to inhibiting cell apoptosis. However, the influence of nodal expression on other pathways involved in RCC growth requires investigation in order to fully understand these mechanisms.

\section{Discussion}

The early symptoms of RCC are insidious, and approximately $30-50 \%$ of RCC cases lack early clinical manifestations (1). In the majority of cases, by the time a patient exhibits three principle symptoms (hematuria, flank pain and palpable abdominal mass), they are in the advanced phase of RCC, and at this stage approximately $30 \%$ of patients will have developed tumor metastasis (2-3). Multi-drug resistance genes may be expressed by RCC cells that are insensitive to chemotherapy or radiotherapy. The efficacy of immunotherapy for RCC remains unclear, and radical nephrectomy is still the most common method of treatment for RCC. However, once lymphatic metastasis occurs, the 5 year survival rate is extremely low (5-15\%), even with radical lymphatic node dissection (2-3). Therefore, early diagnosis and treatment of $\mathrm{RCC}$ is important, and the investigation of tumor biomarkers associated with RCC, which have high specificity and high sensitivity has become a research focus in urology.

$\mathrm{hESCs}$ and tumor cells express the morphogenetic protein, nodal. Nodal may therefore be useful for determining pluripotent phenotypes of tumor cells and hESCs, and for controlling the differentiation of embryonic stem cells $(12,27)$. Increased nodal expression in human melanoma cells, breast, colon and testicular cancer has been demonstrated (8-10). hESCs express lefty, which inhibits nodal signaling pathways. In metastatic tumor cells, nodal is expressed and lefty expression is defective. Therefore, in metastatic tumor cells, the nodal signaling pathway is unregulated. The uncontrolled overexpression of the nodal signaling proteins may lead to the development of malignant tumor cells (7,13-14). A previous study demonstrated that inhibition of the nodal signaling pathway in metastatic melanoma cells may reduce cell colony formation and promote the development of the melanoma cell low-plasticity phenotype (12), reducing tumor inducibility to $\sim 30 \%$ (27). In the present study, the expression of nodal in RCC cells was high, compared with that of adjacent non-tumor cells. However, the expression of lefty in RCC was significantly decreased compared with that of the adjacent non-tumor cells.

Numerous studies have demonstrated that nodal may promote tumor growth, whereas lefty is capable of inhibiting tumor growth (21-26). De Silva et al (30) demonstrated that nodal may promote the tumorous growth of glioblastoma cells, which is mediated by ALK4, ALK7 and smad3 proteins. Cavallari et al (25) demonstrated that lefty A is capable of inhibiting the nodal signaling pathway in human liver stem cells, thereby suppressing tumor cell growth in a similar manner to that observed in hESCs.

The results of the present study suggested that nodal expression may activate the smad and ERK1/2 pathways and promote the growth of RCC. The inhibition of smad3 and the addition of an ERK1/2 pathway inhibitor only partially reduced the capability of nodal expression to promote RCC cell proliferation and invasion, and inhibit cell apoptosis. Lawrence et al (23) demonstrated that recombinant human nodal expression triggered downstream smad2 phosphorylation in DU145 and LNCaP cell lines, and that the stable transfection of pre-pro-nodal enhanced the growth of LNCaP cells in Matrigel and soft agar. Nodal may inhibit androgen receptor signaling, reducing the activity of a prostrate specific antigen promoter and downregulating the endogenous expression of androgen-regulated genes. To the best of our knowledge, there have been limited studies on the influence of nodal and lefty expression on tumor growth. Nodal may promote the growth of RCC by activating the smad and ERK1/2 pathways. These findings provide a basis for further investigations into the association between nodal expression and tumor growth. The results of the present study may therefore be useful for developing novel biomarkers for tumor diagnosis and suggest a potential target gene for the treatment of RCC.

\section{References}

1. Villa G and Hernández-Pastor LJ: Budget impact analysis of first-line treatment with pazopanib for advanced renal cell carcinoma in Spain. BMC Cancer 13: 399, 2013.

2. Nguyen MM, Gill IS and Ellison LM: The evolving presentation of renal carcinoma in the United States: trends from the Surveillance, Epidemiology, and End Results program. J Urol 176: 2397-2400; discussion 2400, 2006.

3. Stafford HS, Saltzstein SL, Shimasaki S, Sanders C, Downs TM and Sadler GR: Racial/ethnic and gender disparities in renal cell carcinoma incidence and survival. J Urol 179: 1704-1708, 2008.

4. Schier AF: Nodal signaling in vertebrate development. Annu Rev Cell Dev Biol 19: 589-621, 2003.

5. Quail DF, Siegers GM, Jewer $M$ and Postovit LM: Nodal signalling in embryogenesis and tumourigenesis. Int J Biochem Cell Biol 45: 885-898, 2013. 
6. Tabibzadeh S and Hemmati-Brivanlou A: Lefty at the crossroads of 'stemness' and differentiative events. Stem Cells 24 1998-2006, 2006.

7. Chen $\mathrm{C}$ and Shen MM: Two modes by which Lefty proteins inhibit nodal signaling. Curr Biol 14: 618-624, 2004

8. Strizzi L, Hardy KM, Kirsammer GT, Gerami P and Hendrix MJ: Embryonic signaling in melanoma: potential for diagnosis and therapy. Lab Invest 91: 819-824, 2011.

9. Strizzi L, Postovit LM, Margaryan NV, et al: Nodal as a biomarker for melanoma progression and a new therapeutic target for clinical intervention. Expert Rev Dermatol 4: 67-78, 2009.

10. Strizzi L, Hardy KM, Margaryan NV, et al: Potential for the embryonic morphogen Nodal as a prognostic and predictive biomarker in breast cancer. Breast Cancer Res 14: R75, 2012.

11. McAllister JC, Zhan Q, Weishaupt C, Hsu MY and Murphy GF The embryonic morphogen, Nodal, is associated with channel-like structures in human malignant melanoma xenografts. J Cutan Pathol 37 (Suppl 1): 19-25, 2010.

12. Postovit LM, Margaryan NV, Seftor EA and Hendrix MJ: Role of nodal signaling and the microenvironment underlying melanoma plasticity. Pigment Cell Melanoma Res 21: 348-357, 2008.

13. Hendrix MJ, Seftor EA, Seftor RE, Kasemeier-Kulesa J, Kulesa PM and Postovit LM: Reprogramming metastatic tumour cells with embryonic microenvironments. Nat Rev Cancer 7 : 246-255, 2007.

14. Postovit LM, Margaryan NV, Seftor EA, et al: Human embryonic stem cellmicroenvironment suppresses the tumorigenic phenotype of aggressive cancer cells. Proc Natl Acad Sci USA 105: 4329-4334, 2008.

15. Cucina A, Biava PM, D'Anselmi F, et al: Zebrafish embryo proteins induce apoptosis in human colon cancer cells (Caco2) Apoptosis 11: 1617-1628, 2006.

16. Lee LM, Seftor EA, Bonde G, Cornell RA and Hendrix MJ: The fate of human malignant melanoma cells transplanted into zebrafish embryos: assessment of migration and cell division in the absence of tumor formation. Dev Dyn 233 1560-1570, 2005

17. Ebele JN, Sauter G, Epstein JI and Sesterhenn IA (eds): Pathology and Genetics of Tumours of the Urinary System and Male Genital Organs. $1^{\text {st }}$ edition. International Agency for Research on Cancer Publications Collection, Lyon, 2004.
18. Edge SB, Byrd DR, Compton CC, Fritz AG, Greene FL, Trotti A (eds): AJCC Cancer Staging Manual. 7th edition. Springer, New York, NY, 2010.

19. Nadeem L, Munir S, Fu G, et al: Nodal signals through activin receptor-like kinase 7 to inhibit trophoblast migration and invasion: implication in the pathogenesis of preeclampsia. Am J Pathol 178: 1177-1189, 2011.

20. Kobayashi T, Liu X, Wen FQ, et al: Smad3 mediates TGF-beta1-induced collagen gel contraction by human lung fibroblasts. Biochem Biophys Res Commun 339: 290-295, 2006.

21. Papageorgiou I, Nicholls PK, Wang F, et al: Expression of nodal signalling components in cycling human endometrium and in endometrial cancer. Reprod Biol Endocrinol 7: 122, 2009.

22. Lonardo E, Hermann PC, Mueller MT, et al: Nodal/Activin signaling drives self-renewal and tumorigenicity of pancreatic cancer stem cells and provides a target for combined drug therapy. Cell Stem Cell 9: 433-446, 2011.

23. Lawrence MG, Margaryan NV, Loessner D, et al: Reactivation of embryonic nodal signaling is associated with tumor progression and promotes the growth of prostate cancer cells. Prostate 71: 1198-1209, 2011.

24. Strizzi L, Postovit LM, Margaryan NV, et al: Emerging roles of nodal and Cripto-1: from embryogenesis to breast cancer progression. Breast Dis 29: 91-103, 2008.

25. Cavallari C, Fonsato V, Herrera MB, Bruno S, Tetta C and Camussi G: Role of Lefty in the anti tumor activity of human adult liver stem cells. Oncogene 32: 819-826, 2013.

26. Saito A, Ochiai H, Okada S, Miyata N and Azuma T: Suppression of Lefty expression in induced pluripotent cancer cells. FASEB J 27: 2165-2174, 2013.

27. Topczewska JM, Postovit LM, Margaryan NV, et al: Embryonic and tumorigenic pathways converge via Nodal signaling: role in melanoma aggressiveness. Nat Med 12: 925-932, 2006.

28. Hardy KM, Kirschmann DA, Seftor EA, et al: Regulation of the embryonic morphogen Nodal by Notch4 facilitates manifestation of the aggressive melanoma phenotype. Cancer Res 70: 10340-10350, 2010.

29. Miyata N, Azuma T, Hozawa S, et al: Transforming growth factor $\beta$ and Ras/MEK/ERK signaling regulate the expression level of a novel tumor suppressor Lefty. Pancreas 41: 745-752, 2012.

30. De Silva T, Ye G,Liang YY,Fu G, Xu G and Peng C: Nodal promotes glioblastoma cell growth. Front Endocrinol (Lausanne) 3: 59, 2012. 